\title{
Groups with infinitely many ends are not fraction groups
}

\author{
Dawid Kielak ${ }^{1}$
}

\begin{abstract}
We show that any finitely generated group $F$ with infinitely many ends is not a group of fractions of any finitely generated proper subsemigroup $P$, that is $F$ cannot be expressed as a product $P P^{-1}$. In particular this solves a conjecture of Navas in the positive. As a corollary we obtain a new proof of the fact that finitely generated free groups do not admit isolated left-invariant orderings.
\end{abstract}

Mathematics Subject Classification (2010). Primary 20F65; Secondary 20F60.

Keywords. Groups with infinitely many ends, groups of fractions, isolated orderings.

\section{Introduction}

The existence of a left-invariant order on a group $G$ is equivalent to the existence of a positive cone $P \subset G$, that is a subsemigroup such that $G$ can be written as a disjoint union $G=\{1\} \sqcup P \sqcup P^{-1}$. In fact there is a one-to-one correspondence between left-invariant orderings and such positive cones.

In this note we prove that whenever a finitely generated group $F$ with infinitely many ends can be written as $F=P P^{-1}$, where $P$ is a finitely generated subsemigroup of $F$, then $P=F$. Our result answers a question of Navas, who conjectured that finitely generated free groups are not groups of fractions of finitely generated subsemigroups $P$ with $P \cap P^{-1}=\emptyset$.

As an application we obtain a new proof of the fact that the space of leftinvariant orderings of a finitely generated free group (endowed with the Chabauty topology) does not have isolated points. This result follows from the work of McCleary [2], but appears in this form for the first time in the work of Navas [3]. It is worth noting that our proof is the first geometric one.

\footnotetext{
${ }^{1}$ Author supported by the ERC Grant Nb. 10160104.
} 
We also deduce that the left-orderings of finitely generated groups with infinitely many ends do not have finitely generated positive cones. This was already known for free products of left-orderable groups by the work of Rivas [4].

Our theorem complements a folklore result stating that whenever $\mathcal{S}$ is a finite generating set for a group $G$, and $G$ does not contain a free subsemigroup, then $G$ is a group of fractions of $P$, the semigroup generated by $\delta$.

We should note here that finitely generated groups with infinitely many ends have been classified by Stallings [5, 6]. They are precisely those fundamental groups of non-trivial graphs of groups with exactly one edge and a finite edge group, which are finitely generated and not virtually cyclic.

Acknowledgments. The author wishes to thank Andrés Navas for introducing him to this problem, and for his many comments. He also wishes to thank Thomas Haettel and Ursula Hamenstädt for many helpful conversations, and the referee for pointing out ways of significantly improving the presentation of this note.

\section{The result}

In the following we will use $X$ to denote the (right) Cayley graph of a finitely generated group $F$ with respect to some finite generating set. We will identify $F$ with vertices of $X$, and use $d$ to denote the standard metric on the Cayley graph $X$. The isometric left-action of $F$ on $X$ and its subsets will be denoted by left multiplication. The notation $B(x, \xi)$ will stand for the closed ball centred at $x$ of radius $\xi$.

We will assume that $F$ has infinitely many ends, and so there will exist a constant $\kappa$ such that the ball $B=B(1, \kappa)$ disconnects $X$ into a space with at least 3 infinite components. We will use $S$ to denote the set of vertices of $B$.

Definition 2.1. We say that $A \subset X$ is a shoot if and only if there exists $w \in F$ such that $A$ is a connected component of $X \backslash w B$. We say that $w B$ bounds the shoot.

Lemma 2.2. Let $A$ be an infinite shoot bounded by $B$. Then there exists $w \in F$ such that $w(X \backslash A) \subseteq A$ and $w^{-1}(X \backslash A) \subseteq A$.

Proof. Note that the ball $B(1,2 \kappa)$ is finite, since $X$ is locally finite. Since $F$ has infinitely many ends and $A$ is infinite, there exists $\lambda$ such that

$$
L=\{x \in F \mid d(1, x)=\lambda\} \cap A
$$


has more than $|B(1,2 \kappa)|$ elements. Take $l \in L$. The cardinality of $L$ guarantees that there exists $l^{\prime} \in L$ such that $l^{\prime} l^{-1} \notin B(1,2 \kappa)$.

Let $w=l^{\prime} l^{-1}$. Observe that

$$
d\left(w, l^{\prime}\right)=d\left(l^{\prime} l^{-1}, l^{\prime}\right)=d(1, l)=\lambda
$$

Consider a shortest path between $w$ and $l^{\prime}$. If it lies entirely in $A$, then in particular so does $w$. If not, then it must contain some point $b \in B$, since $B$ bounds $A$. Now we have

$$
\lambda=d\left(w, l^{\prime}\right)=d(w, b)+d\left(b, l^{\prime}\right) \geqslant d(w, b)+\lambda-\kappa
$$

which implies that $d(w, b) \leqslant \kappa$, and hence that $w \in B(1,2 \kappa)$, which is a contradiction. We have thus established that $w \in A \backslash B(1,2 \kappa)$, and therefore that $w B \subset A$.

Note that $w^{-1}=l l^{\prime-1} \notin B(1,2 \kappa)$ enjoys the same properties as $w$, and so we immediately conclude that $w^{-1} B \subset A$, or equivalently that $B \subset w A$.

Since $w B$ and $B$ are disjoint, every shoot bounded by $w B$ either contains $B$ or is disjoint from it. Clearly, there is a unique shoot bounded by $w B$ containing $B$, and we have already shown that it is $w A$. Each of the other shoots bounded by $w B$ lies in a single shoot bounded by $B$, namely in the shoot bounded by $B$ which contains $w B$. But we have already seen that this is $A$. We are left with the conclusion that $w(X \backslash A) \subset A$, and our proof is finished by making the analogous observations for $w^{-1}$.

We are now ready for the main result.

Theorem 2.3. Let $P$ be a finitely generated subsemigroup of a finitely generated group $F$ with infinitely many ends. If $P P^{-1}=F$, then $P=F$.

Proof. For ease of notation we will refer to the elements of $P$ as positive, and to the elements of $P^{-1}$ as negative.

We first note that any finite generating set of $P$ is a generating set for $F$. Let $X$ be the Cayley graph of $F$ with respect to some such generating set. Note that this allows us to view generators of $P$ as positive edges of $X$, and hence any positive element $p \in P$ is realised by a positive path between 1 and the vertex $p$ in $X$.

We will use the notation $\kappa, B$ and $S$ as defined above.

$\mathrm{S}_{\text {TEP }} 1$. We claim that $S\left(P^{-1} \cup\{1\}\right)=F$.

If $P$ intersects each ball $B(x, \kappa)=x B$ then each $x \in F$ is a concatenation of an element of $P$ (namely any positive path from 1 to $x B$ ) with an element in $S$ 
(connecting the end of the positive path to the centre of the ball). Thus we have $x \in P S$, and our claim follows by taking inverses.

Let us now suppose that there exists an $x \in F$ such that

$$
P \cap x B=\emptyset
$$

Let $A_{0}$ denote an infinite shoot bounded by $x B$ such that $1 \notin A_{0}$.

Let $z \in F \backslash S$ be any element, and let $A$ be the shoot bounded by $B$ containing $z$. We claim that there exists $y \in F$ such that $y A \subseteq A_{0}$.

There are two cases we need to consider. The first one occurs when

$$
x A \subseteq A_{0}
$$

in which case we take $y=x$. The other one (illustrated in Figure 1) occurs when $x A \nsubseteq A_{0}$, that is when $x A$ is a shoot bounded by $x B$ other than $A_{0}$. Lemma 2.2 applied to $x^{-1} A_{0}$ gives us an element $w \in F$ such that $w\left(X \backslash x^{-1} A_{0}\right) \subseteq x^{-1} A_{0}$. So $y=x w$ satisfies

$$
y A=x w A \subseteq x x^{-1} A_{0}=A_{0}
$$

and so we have proven the claim.

Now, since $y z \in F=P P^{-1}$, we can write $y z=p q$, where $p$ is positive and $q$ is negative. Since there are no positive elements in $x B$ by assumption, we see that $p \notin A_{0}$, and therefore $q$ is a negative path connecting a vertex $p \in X \backslash A_{0}$ to $y z \in y A \subseteq A_{0}$. The shoot $y A$ is bounded by $y B$ and contained in $A_{0}$, hence any path from $X \backslash A_{0}$ to $y A$ has to cross $y B$. This is in particular true for $q$, so there is a negative path (a terminal subpath of $q$ ) from some vertex of $y B$ to $y z$, and hence from a vertex of $B$ to $z$ (after translating by $y^{-1}$ ). In the group language we have thus shown that $z \in S P^{-1}$, and so

$$
F \backslash S \subseteq S P^{-1}
$$

But clearly $S \subset S\left(P^{-1} \cup\{1\}\right)$, and so we have proven the claim of Step 1 .

SteP 2. We claim that $P=F$.

We have established above that $S\left(P^{-1} \cup\{1\}\right)=F$, with $S$ being finite. Let $Q$ be a minimal (with respect to cardinality) finite subset of $F$ such that

$$
Q\left(P^{-1} \cup\{1\}\right)=F .
$$

Suppose that there exist distinct $q, q^{\prime} \in Q$. Then $q^{-1} q^{\prime} \in F=P P^{-1}$, and so $q^{-1} q^{\prime}=a b^{-1}$ with $a, b \in P$. Hence

$$
q, q^{\prime} \in q a P^{-1}
$$


and therefore we could replace $Q$ by $(Q \cup\{q a\}) \backslash\left\{q, q^{\prime}\right\}$ of smaller cardinality. This shows that $|Q|=1$. Without loss of generality we can take $Q=\{1\}$, and thence get

$$
P^{-1} \cup\{1\}=F
$$

Now let $f \in F \backslash\{1\}$. We have $f, f^{-1} \in P^{-1}$, and since $P^{-1}$ is a semigroup, also $1=f f^{-1} \in P^{-1}$. So $P^{-1}=F$. Taking an inverse concludes the theorem.

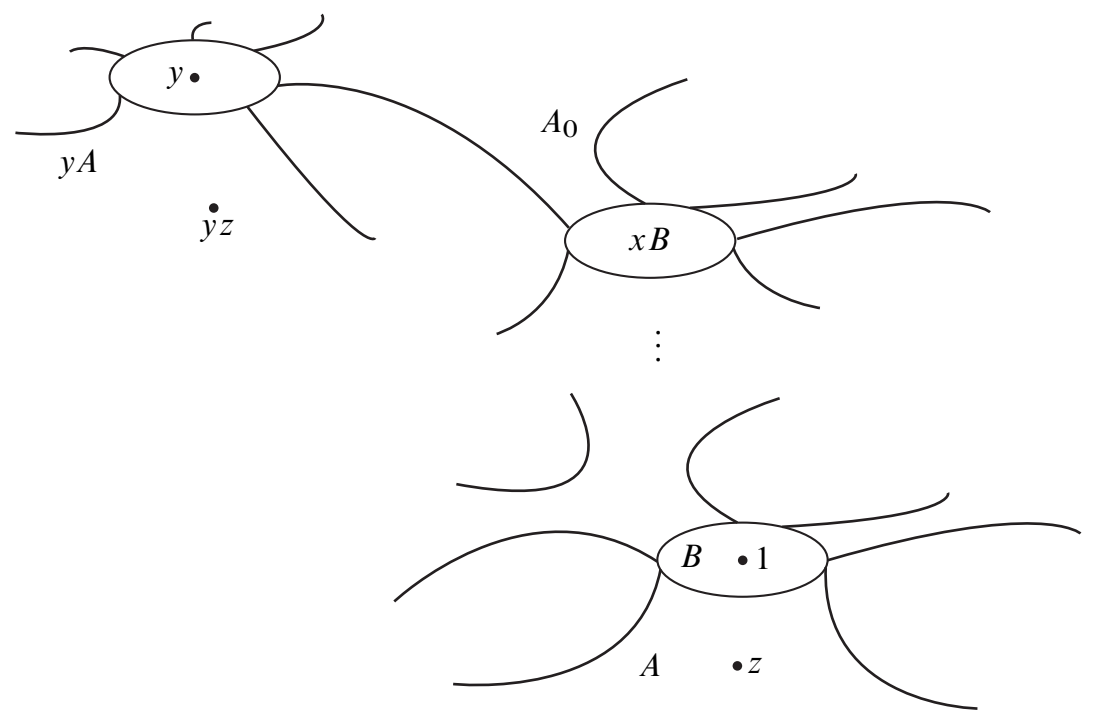

Figure 1. Step 1 of the theorem.

We now easily deduce the following.

Corollary 2.4. Let $F$ be a finitely generated group with infinitely many ends. Then $F$ does not allow a left-invariant ordering with a finitely generated positive cone.

Proof. Let $P$ be the positive cone of a left-invariant ordering of $F$. Then

$$
F=P \cup P^{-1} \cup\{1\}
$$

and so in particular $F=P P^{-1}$. But also $P \cap P^{-1}=\emptyset$, and so $P \neq F$. Now the contrapositive of Theorem 2.3 tells us that $P$ is not finitely generated. 
The statement above follows from the work of Rivas [4], since left-orderable groups are torsion-free, and so they have infinitely many ends only when they are free products.

We also get the following corollary.

Corollary 2.5. The space of left-invariant orderings on any finitely generated free group has no isolated points.

Proof. Let $P$ be the positive cone of an isolated ordering of $F$, a finitely generated free group. By above, $P$ is not finitely generated.

The order defined by $P$ is isolated, and so there exists a finite set $S \subset F$ such that whenever we have another positive cone of an ordering $P^{\prime}$ such that $P \cap S=$ $P^{\prime} \cap S$, then $P=P^{\prime}$. However the work of Smith and Clay [1, Theorem E] allows us to construct an order (in fact infinitely many such orders) whose positive cone $P^{\prime}$ satisfies $P \cap S=P^{\prime} \cap S$, but such that $P \neq P^{\prime}$. This is a contradiction.

Added in proof. From the main theorem one can also easily deduce that groups with finite Garside structures have at most two ends.

\section{References}

[1] A. Clay and L. H. Smith, Corrigendum to: "On ordering free groups" [J. Symbolic Comput. 40 (2005) 1285-1290.] J. Symbolic Comput. 44 (2009), no. 10, 1529-1532. Zbl 2543435 MR 2543435

[2] S. H. McCleary, Free lattice-ordered groups represented as $o-2$ transitive $l$-permutation groups. Trans. Amer. Math. Soc. 290 (1985), no. 1, 69-79. Zbl 0546.06013 MR 0787955

[3] A. Navas, On the dynamics of (left) orderable groups. Ann. Inst. Fourier (Grenoble) 60 (2010), no. 5, 1685-1740. Zbl 05822118 MR 2766228

[4] C. Rivas, Left-orderings on free products of groups. J. Algebra 350 (2012), 318-329. Zbl 1261.06021 MR 2859890

[5] J. R. Stallings, On torsion-free groups with infinitely many ends. Ann. of Math. (2) 88 (1968), 312-334. Zbl 0238.20036 MR 0228573

[6] J. R. Stallings, Group theory and three-dimensional manifolds. A J. K. Whittemore Lecture in Mathematics given at Yale University, 1969. Yale Mathematical Monographs, 4. Yale University Press, New Haven, CT, and London, 1971. Zbl 0241.57001 MR 0415622 
Received May 7, 2013

Dawid Kielak, Mathematisches Institut der Universität Bonn, Endenicher Allee 60, D-53115 Bonn, Germany

e-mail:kielak@math.uni-bonn.de 